\title{
Prevalencia de leptospirosis en perros vagos capturados en la ciudad de Temuco, 2011
}

\author{
Christian Tuemmers, Carlos Lüders, Claudio Rojas, Michel Serri, Rodrigo Espinoza y Carolina Castillo
}

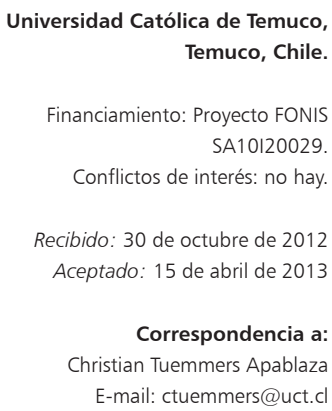

Universidad Católica de Temuco, Temuco, Chile.

Financiamiento: Proyecto FONIS SA10120029

Conflictos de interés: no hay.

Recibido: 30 de octubre de 2012 Aceptado: 15 de abril de 2013

Correspondencia a: Christian Tuemmers Apablaza E-mail: ctuemmers@uct.c

\section{Prevalence of leptospirosis in vague dogs captured in Temuco city, 2011}

Background: Leptospirosis, a bacterial disease of worldwide distribution, affects various animals and is considered a zoonosis. It can be transmitted directly or indirectly, mainly through contact with the carrier's urine and entering the body through mucous membranes or skin. In the city of Temuco, there are no epidemiological studies of canine leptospirosis and the country data are scarce. Objective: To determine the prevalence of leptospirosis in stray dogs of the city of Temuco. Material and Methods: In a cross- sectional study, 400 dogs admitted to Temuco Kennel during the year 2011 were sampled. Blood samples were analyzed using a modified commercial ELISA kit. Results: The prevalence of leptospirosis was $21.3 \%$. Positive cases were concentrated in dogs 5 to 8 years of age, independent of gender. Discussion: The high prevalence found demonstrates the need for further studies to better understand the epidemiology of the disease and to establish prevention and control measures.

Key words: Leptospirosis, zoonosis, stray dog, Weil disease.

Palabras clave: Leptospirosis, zoonosis, perro vago, enfermedad de Weil.

\section{Introducción}

$\mathrm{L}$ a leptospirosis es una enfermedad de distribución mundial y merece especial mención dentro de las zoonosis $^{1-5}$, siendo reconocida prácticamente en cualquier sitio donde exista convivencia con animales ${ }^{6}$. Fue descrita por primera vez en el hombre en $1886^{7,8}$ y en caninos en el año $1899^{9}$. Esta patología es causada por bacterias patógenas del género Leptospira ${ }^{2} \mathrm{y}$, si no es tratada oportunamente, puede llegar a ser mortal ${ }^{10}$. A pesar de esto, es considerada una enfermedad de baja mortalidad, pero de alta morbilidad ${ }^{11}$.

Afecta a diversos mamíferos como bovinos, equinos, caninos, roedores y humanos, entre otros ${ }^{12}$. Los animales son considerados hospederos de mantenimiento de la enfermedad ${ }^{13} \mathrm{y}$ los distintos serovares tienen predilección por algunas especies ${ }^{8,12}$, entre los que podemos nombrar: L. canicola asociada al perro, L. hardjo para bovinos, L. pomona para bovinos y cerdos, L. bratislava para los $\operatorname{cerdos}^{5,13}$ y L. icterohaemorrhagiae a roedores y perros, siendo ésta la que produce con mayor frecuencia infección grave en humanos ${ }^{12}$.

La leptospirosis se puede transmitir directa o indirectamente, entre animales, o de los animales a las personas. La forma directa se produce al entrar en contacto con sangre, tejidos u órganos de animales infectados ${ }^{14}$, mientras que la forma indirecta es la más frecuente, donde el agua juega un papel primordial ${ }^{1}$, ya que las leptospiras provenientes de terrenos o agua contaminada con orina de animales enfermos ingresan al organismo a través de la mucosa oral, conjuntival, nasal o genital; así mismo, a través de la piel con laceraciones o reblandecida por la humedad ${ }^{15,16}$. Las tasas de transmisión son muy elevadas ya que sólo 10 microorganismos son necesarios para causar la enfermedad ${ }^{9}$.

Esta afección es considerada una zoonosis de riesgo profesional, siendo los más afectados los médicos veterinarios, agricultores y empleados de mataderos ${ }^{17}$. Pese a esto, actualmente existe una disminución del contagio de leptospirosis por riesgo ocupacional y ha aumentado el riesgo recreacional, especialmente asociado al turismo aventura, donde un gran número de veraneantes se bañan en aguas estancadas en la época estival ${ }^{8}$.

Esta enfermedad constituye un importante problema emergente en la Salud Pública ${ }^{18}$, debido a la incidencia cada vez mayor, tanto en países en vías de desarrollo como en los países desarrollados ${ }^{19}$; es más común en áreas tropicales y subtropicales con altos índices de precipitación ${ }^{2}$.

En cuanto al agente etiológico, Leptospira sp es muy sensible a la desecación, a la exposición directa a los rayos solares ${ }^{7} \mathrm{y}$ a los cambios de $\mathrm{pH}$ (vive en $\mathrm{pH}$ entre 6 y 8), pero sobrevive hasta tres meses en suelos húmedos, aumentando este tiempo en aguas estancadas ${ }^{7,20}$. Por esto, la tasa de incidencia de la leptospirosis se incrementa en épocas lluviosas en regiones de clima cálido ${ }^{13}$.

La incidencia anual estimada en el ser humano varía de 0,1 a 1 caso por 100.000 individuos en zonas templadas, de 10-100 casos por 100.000 individuos en climas húmedos tropicales y cuando se producen brotes o epidemias la incidencia puede alcanzar más de 100 casos por 100.000 individuos ${ }^{2}$.

Los focos más importantes de la enfermedad se en- 
cuentran en el Caribe, América Latina, India, sur este de Asia, Oceanía e incluso en la Unión Europea o Japón²1,22.

Diversos autores señalan que el cambio climático favorecerá el aumento en la incidencia mundial de leptospirosis ${ }^{23}$, ya que provocará situaciones climáticas extremas como inundaciones, cuando el área de la salud y sus recursos serán limitados si no se toman los recaudos sanitarios con la debida anticipación ${ }^{24}$.

En Chile, la leptospirosis está presente y su incidencia se conoce a partir del año 2000, cuando fue incorporada como enfermedad de notificación obligatoria a través del diagnóstico de laboratorio ${ }^{25}$, descrito en el reglamento $\mathrm{N}^{\mathrm{o}} 712$ (actual DS 158), emanado por el MINSAL, bajo la responsabilidad del Instituto de Salud Pública. Sin embargo, no se han realizado estudios sistemáticos que den a conocer la situación real que afecta a la población humana a lo largo del territorio nacional. A diferencia de estudios en animales, donde sí se han pesquisado, principalmente en el sur del país ${ }^{26}$, éstos no son suficientes para demostrar el estatus zoosanitario en cuanto a la prevalencia de leptospirosis.

Existen estudios relevantes sobre prevalencia de leptospirosis humana en Chile, como el realizado por Perret y cols. ${ }^{25}$, quienes detectaron $3,3 \%$ de casos positivos a leptospirosis en una población de riesgo en la Región Metropolitana. Por otro lado, Arias y cols. ${ }^{27}$, analizaron un brote epidémico de leptospirosis acaecido en niños de Linares, en el que de 182 niños expuestos, 90 fueron positivos a leptospirosis. Riedemann y Zamora ${ }^{28}$, realizaron un importante estudio en 239 campesinos del sur de Chile, expresando una positividad de 19,6\%.

La prevalencia de leptospirosis animal ha sido mayormente estudiada, especialmente, en algunas regiones sureñas de Chile ${ }^{8}$. Silva y Riedemann ${ }^{18}$, en la ciudad de Valdivia, obtuvieron una prevalencia de $14,8 \%$. Por otra parte, Lobos $^{29}$ detectó una positividad de $30 \%$ en perros vagos de la ciudad de Chillán. Sothers ${ }^{30}$, obtuvo $31,05 \%$ de prevalencia en perros de distintas zonas de la ciudad de Chillán, mientras que García ${ }^{31}$ encontró 38,3\% de positividad en perros atendidos en la Clínica Veterinaria de la Universidad de Concepción, en Chillán. Por otro lado, el estudio realizado por Zamora, Kruze y Riedemann ${ }^{32}$, entregó cifras de prevalencia de leptospirosis de 41,9\% en zonas rurales del sur de Chile.

Por lo anteriormente mencionado se fijó como objetivo: determinar la prevalencia de leptospirosis en perros vagos capturados durante el año 2011 en la ciudad de Temuco e ingresados al Canil Temuco.

\section{Material y Métodos}

Este estudio contó con el auspicio y colaboración entre la SEREMI de Salud Araucanía Sur, Municipalidad de Temuco y Universidad Católica de Temuco.
Este proyecto correspondió a un estudio de carácter cuantitativo y de corte transversal y se desarrolló en un período de 18 meses, procurando muestras de perros vagos de la ciudad de Temuco ingresados al Canil Temuco, durante el año 2011. Éste se realizó por un método no probabilístico intencionado y se seleccionaron los animales que cumplían los criterios de haber sido ingresados como máximo durante las $48 \mathrm{~h}$ previas a la toma de muestra y ser considerados vagos. Estos perros, capturados por personal de la Municipalidad de Temuco, deambulaban libremente por las calles de la ciudad, plazas, campamentos y el vertedero municipal, sin ningún tipo de restricción. Además se encontraban en estrecho contacto con otros canes vagos y posiblemente también con animales domésticos de hábitos callejeros.

Para el cálculo del tamaño de la muestra se empleó la fórmula sugerida por Thrusfield (1995) ${ }^{33}$.

$$
\mathrm{n}=1,962 \operatorname{Pexp}(1-\mathrm{Pexp}) / \mathrm{d} 2
$$

La prevalencia estimada fue fijada en $50 \%$, ya que se desconoce la prevalencia de la zoonosis, estableciendo un IC de $95 \%$ y obteniéndose un tamaño de muestra de 384 perros. Sin embargo, el número de perros vagos establecido fue de 400, a los efectos de salvaguardar las posibles exclusiones según los criterios fijados.

El procedimiento de toma de muestras e identificación de agentes infecciosos se realizó bajo normas de bioseguridad, manteniendo la higiene adecuada y resguardando la integridad física, con la utilización de bozales y/o tranquilizantes en los caninos, en caso que fuese necesario.

Las muestras fueron obtenidas mediante punción de la vena cefálica, quedando como segunda opción la vena safena. Una vez extraídas las muestras fueron depositadas en un tubo Vacutainer estéril sin anticoagulante, se rotularon con el número de identificación del canino y se trasladaron refrigeradas al laboratorio, para luego de su coagulación ser centrifugadas durante 5 min a 3.500 rpm. Los sueros obtenidos se congelaron a $-20^{\circ} \mathrm{C}$ hasta el momento de su análisis.

Para el análisis diagnóstico se utilizó el kit comercial serológico: ImmunoComb Canine Leptospira Antibody Test $\mathrm{Kit}^{\circledR}$. Esta prueba corresponde a una modificación del método ELISA (inmunoensayo enzimático) y detecta los títulos de anticuerpos presentes en el suero o la sangre entera, obteniéndose resultados en $20 \mathrm{~min}$. Contiene una mezcla de serovares de L. interrogans: L. icterohaemorrhagiae, L. canicola, L. pomona y L. grippotyphosa. El kit diagnóstico no identifica los serovares que afectan al animal; sin embargo, permite conocer el grado de positividad de la reacción, que puede ser baja, moderada o alta, identificándose en niveles de $\mathrm{S} 0$ a S6, respectivamente. Estos resultados son comparables con la prueba de MAT (microaglutinación en tubo), es decir, muestras con resultados entre S1 y S2 son positivamente bajos, y equivalen 
Figura 1. Distribución porcentual de caninos positivos y negativos a leptospirosis, año 2011.

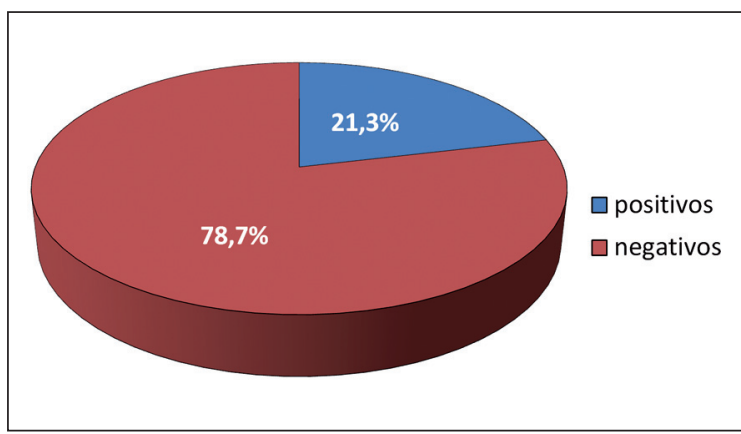

Tabla 2. Muestras caninas clasificadas por sexo, año 2011

\begin{tabular}{|clcccc|}
\hline Válidos & Machos & $\begin{array}{c}\text { Frecuencia } \\
\text { positivos }\end{array}$ & $\begin{array}{c}\text { Frecuencia } \\
\text { negativos }\end{array}$ & $\begin{array}{c}\text { Frecuencia } \\
\text { total }\end{array}$ & $\begin{array}{c}\% \text { positivos } \\
\text { por sexo }\end{array}$ \\
& 37 & 135 & 172 & 21,5 \\
& Hembras & 48 & 180 & 228 & 21,1 \\
\hline & Total & 85 & 315 & 400 & \\
\hline
\end{tabular}

Figura 2. Muestras caninas positivas y negativas clasificadas por sexo, año 2011.

Figura 3. Muestras caninas positivas y negativas clasificadas por grupo etario, año 2011.

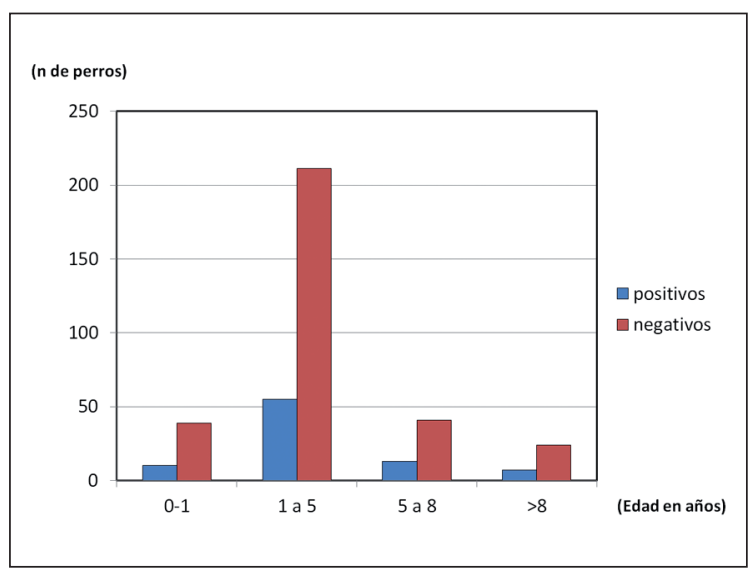

\begin{tabular}{|ccccc|}
\hline \multicolumn{5}{|c|}{ Tabla 1. Seroprevalencia de leptospirosis en perros } \\
vagos de Temuco, año 2011
\end{tabular}

a títulos de 1:100 o 1:200 en la MAT, mientras que S3 indica positividad moderada, equivalente a 1:400 títulos en la MAT y finalmente resultados iguales o superior a S4 son altos, sobre 1:400 títulos ${ }^{34}$.

El análisis estadístico de los resultados se realizó mediante el programa estadístico SPSS, aplicando estadística descriptiva y $\chi^{2}$ para establecer si los animales positivos presentaban independencia de la edad y el sexo, a un nivel de significación de $5 \%$.

\section{Resultados}

De la población estudiada, 172 perros fueron machos y 228 fueron hembras, representando 43 y $57 \%$ de la población, respectivamente. El rango de edad de los animales estudiados fue de 2 meses a 12 años con una mediana de 3 años.

De 400 animales evaluados, 85 resultaron positivos, calculándose la prevalencia de leptospirosis en $21,3 \%$ (Tabla 1, Figura 1).

Del total de machos estudiados, $21,5 \%$ (n: 37 ) resultaron positivos, mientras que $21,2 \%$ (n: 48 ) del total de hembras, presentaron leptospirosis, no encontrándose diferencias significativas entre ambos, $\mathrm{P}>0,05$ (Tabla 2, Figura 2).

Respecto a la edad, se observaron diferencias donde la mayor seropositividad se presentó en animales de entre 5 y 8 años de edad, con $24,07 \%$, mientras la menor positividad se observó en cachorros (menores a 1 año) con 20,4\% (Tabla 3, Figura 3).

\section{Tabla 3. Muestras caninas clasificadas por grupo etario,} año 2011

\begin{tabular}{|llrrr|}
\hline \multicolumn{2}{|c}{ Edad (años) } & \multicolumn{2}{c}{ Diagnóstico } & Total \\
& & \multicolumn{1}{c}{$(+)$} & $(-)$ & \\
$0-1$ & (Cachorros) & $10(20,41)$ & $39(79,5)$ & 49 \\
1 a 5 & (Jóvenes) & $55(20,68)$ & $211(79,3)$ & 266 \\
5 a 8 & (Adultos) & $13(24,07)$ & $41(75,9)$ & 54 \\
$>8$ & (Viejos) & $7(22,58)$ & $24(77,4)$ & 31 \\
Total & & $85(21,25)$ & $315(78,7)$ & 400 \\
\hline
\end{tabular}




\section{Discusión}

Para el diagnóstico de la leptospirosis es necesario utilizar métodos sencillos y rápidos de ejecución siendo las técnicas serológicas las de mayor precisión y eficacia para estas investigaciones ${ }^{35}$. El método diagnóstico utilizado en este estudio fue el kit comercial ImmunoComb Canine Leptospira Antibody Test Kit ${ }^{\circledR}$, que corresponde a un test de ELISA modificado, con un alto índice de sensibilidad (98\%) y especificidad (97\%). Esta prueba resultó ser eficaz en simplicidad y rapidez en el análisis de leptospirosis, comparada con la MAT (test de aglutinación microscópica), prueba de referencia y comúnmente utilizada para el diagnóstico de esta patología ${ }^{36}$, presentando una sensibilidad de $90 \%$ y especificidad de $100 \%{ }^{18,37}$. El método diagnóstico utilizado puede provocar diferencias entre investigaciones de un mismo tipo ${ }^{18}$.

En el presente estudio se obtuvo 21,3\% de prevalencia de leptospirosis en perros vagos de la ciudad de Temuco, presentándose la enfermedad principalmente en perros adultos $(24,07 \%)$, coincidiendo con lo informado por la Sociedad Chilena de Infectología Veterinaria (Sochivet) y el Comité de Infecciones Emergentes de la Sociedad Chilena de Infectología ${ }^{38}$. Por otro lado, Tilley, Smith y MacMurray $^{39}$, indican que la enfermedad afecta a perros de cualquier edad y que la incidencia es mayor en caninos machos, contrario a los resultados obtenidos en este estudio.

Los perros, por su capacidad de diseminación de agentes patógenos, tienen gran relevancia en el ciclo de la enfermedad según lo describen distintos autores ${ }^{40,41}$, siendo la calle el factor de riesgo más importante para los caninos. Se ha demostrado que los canes orinan, beben y comen generalmente en los mismos lugares, constituyendo un ambiente notablemente favorable para la transmisión de la leptospirosis; sumando a esto la presencia de roedores como gran reservorio del patógeno ${ }^{42}$, lo que puede explicar la alta prevalencia de leptospirosis obtenida en el estudio.

Si se compara la prevalencia obtenida en la ciudad de Temuco, con otros trabajos realizados en Chile, las diferencias son variables. Lobos ${ }^{29}$, entrega cifras de 30\% de prevalencia en un estudio realizado en 100 perros vagos de la ciudad de Chillán. García ${ }^{31}$, determinó una prevalencia de 38,3\% de leptospirosis en perros urbanos de la ciudad de Chillán. Pineda, López y García ${ }^{43}$, detectaron una positividad de 38,3\% para leptospirosis en perros escogidos al azar de la casuística del Hospital Veterinario de la Universidad de Concepción, sede Chillán. Sothers ${ }^{30}$, comprobó que la prevalencia de leptospirosis en perros callejeros escogidos al azar en la ciudad de Chillán fue de $31,05 \%$. Por otra parte, en Valdivia se determinó una prevalencia de $14,8 \%{ }^{18}$ en 400 perros atendidos en clínicas veterinarias de la ciudad. Muy diferente fue la prevalencia de leptospirosis obtenida en el sector rural de la misma provincia el año 1966 , donde $52,5 \%$ de los perros evaluados fueron positivos ${ }^{44}$.

La misma variabilidad de resultados existe con estudios realizados en el extranjero, como el de Rivera y cols. ${ }^{45}$, quienes encontraron $38,5 \%$ de prevalencia en perros callejeros del norte de Ciudad de México. Por otro lado, Rosas ${ }^{46}$, realizó un estudio en dos albergues caninos, uno en Veracruz y otro en Boca del Río, obteniendo una prevalencia de 4,3\% en ambos albergues. La prevalencia obtenida en perros vagos de Temuco es considerablemente mayor, comparada con los resultados obtenidos en Veracruz y Boca del Río, pero menor en comparación con Ciudad de México. Por otra parte, en una investigación en Colombia, donde se analizaron 900 caninos (con dueño y sin vacunar) de tres municipios de Tolima: Lérida, Mariquita y Piedras se estableció una prevalencia de leptospirosis de 20,2\% y se determinó que existe diferencia estadísticamente significativa en relación al sexo de los animales positivos ${ }^{47}$, lo que difiere con los resultados obtenidos en el presente estudio, donde no se encontraron diferencias estadísticamente significativas entre machos y hembras.

Los resultados demuestran que las prevalencias son variables, debido a las distintas realidades y factores asociados a la persistencia de esta zoonosis en el ambiente, lo cual dificulta la extrapolación entre los distintos lugares geográficos que se han estudiado, obligando a realizar estudios individuales en cada continente, país, región o zona ${ }^{48}$.

Se concluye que la leptospirosis es una zoonosis a considerar y que requiere de un mayor manejo del medio ambiente, control de reservorios y manejo sanitario de las mascotas, requiriéndose una vigilancia epidemiológica activa de la enfermedad para la toma de decisiones oportunas y efectivas por parte de las autoridades.

\section{Resumen}

Introducción: La leptospirosis es una enfermedad bacteriana de distribución mundial, afecta a diversos animales y es considerada una zoonosis. Puede transmitirse de manera directa o indirecta, principalmente por contacto con orina de un animal portador, ingresando al organismo a través de las mucosas o la piel reblandecida por la humedad. En la ciudad de Temuco no existen estudios epidemiológicos de leptospirosis canina y los datos en el país son escasos. Objetivo: Realizar un estudio de corte transversal, para determinar la prevalencia de leptospirosis en perros vagos de la ciudad de Temuco. Material y Métodos: Se procuró muestras de un total de 400 perros ingresados al Canil Temuco durante el año 2011. Se recolectaron muestras de sangre para luego ser analizadas 
mediante un kit comercial de ELISA modificado. Resultados: La prevalencia de leptospirosis en perros vagos de la ciudad de Temuco fue 21,3\%. La mayoría de los casos positivos se concentran en perros de 5 a 8 años de edad e independiente del sexo. Discusión: La alta prevalencia encontrada demuestra la necesidad de mayores estudios tendientes a comprender mejor la epidemiología de la enfermedad y poder establecer medidas de prevención y control que eviten el riesgo de exposición del hombre a esta zoonosis.

\section{Referencias bibliográficas}

1.- Solano A, Boza R, Sáenz E. Leptospirosis en humanos. Rev Costarric Cienc Méd 1996; 17 (2): 41-60.

2.- OMS. Leptospirosis humana: Guía para el diagnóstico, vigilancia y control. 2008 Disponible en: http://www.med.monash.edu.au/ microbiology/staff/adler/guia-esp.pdf (Acceso el 14 de mayo de 2011).

3.- Tabío Y, Palmero Y, Cruz E. Leptospirosis humana y factores de riesgo. Revista Infociencia 2010; 14 (3): 1-11.

4.- Borbolla M, García L, Cadenas Ma, Hernández $\mathrm{R}$, De la Fuente J, Piña $\mathrm{O}$, et al. Leptospirosis durante la contingencia ambiental por inundación en Tabasco 2008. Salud en Tabasco 2009; 15 (2 y 3): 860-7.

5.- Adler B, Lo M, Seemann T, Murray G. Pathogenesis of leptospirosis: The influence of genomics. Vet Microbiol 2011; 153: 73-81.

6.- Macedo J, González J, Márquez M D. Leptospirosis: zoonosis emergente. Informe de un caso. Med Int Mex 2007; 23: 244-7.

7.- Rodríguez G. Estado actual de la leptospirosis. MVZ-Córdoba 2000; 5 (1): 61-3.

8.- Zunino E, Pizarro R. Leptospirosis. Puesta al día. Rev Chilena Infectol 2007; 24 (3): 220-6.

9.- Ford R. Zoonoses: How real the threat? 2010. Disponible en: http://veterinarycalendar. dvm360.com/avhc/Veterinary+team/ Zoonoses-How-real-is-the-threat-Proceedings ArticleStandard/Article/detail/738496 (Acceso el 30 de abril de 2011).

10.- Céspedes M. Leptospirosis: enfermedad zoonótica reemergente. Rev Perú Med Exp Salud Pública 2005; 22 (4): 290-307.

11.- Dubraska V, Díaz C. Manual de ganadería doble propósito: leptospirosis. 2005. Disponible en: http://www.avpa.ula.ve/docuPDFs/libros online/manual-ganaderia/seccion5/articulo4-s5. pdf (Acceso el 1 de mayo de 2012).

12.- Dabanch J. Zoonosis. Rev Chilena Infectol 2003; 20 (1): 47-51

13.- Levett P. Leptospirosis: A forgotten zoonosis? Clin Applied Immunol Rev 2004; 4: 435-48.

14.- MINSAL. Circular de vigilancia y control de leptospirosis, 2009. $\mathrm{N}^{\circ} 3$; circular $\mathrm{N}^{\circ} \mathrm{B} 51 / 10$. Depto. de Epidemiología.

15.- Pizarro J. "Detección y análisis de los principales riesgos profesionales que afectan a médicos veterinarios del área de animales mayores en la provincia de Malleco, IX región de la Araucanía". Memoria de Título de Médico Veterinario. Universidad Católica de Temuco,
Facultad de Acuicultura y Ciencias Veterinarias, Escuela de Medicina Veterinaria, Temuco, Chile. 2004.

16.- Luna A, Moles C, Gavaldón R, Nava V, Salazar G. La leptospirosis canina y su problemática en México. Rev Salud Anim 2008; 30 (1): 1-11.

17.- Roca B. Leptospirosis. Revista de Medicina de la Universidad de Navarra 2006; 50 (2): 3-6.

18.- Silva R F, Riedemann S. Seroprevalencia de leptospirosis canina en perros atendidos en clínicas veterinarias, mediante aglutinación microscópica y comparación con las técnicas de aislamiento e inmunofluorescencia indirecta. Arch Med Vet 2007; 39 (3): 269-74.

19.- Meites E, Jay M, Deresinski S, Shieh W, Zaki S, Tompkins L, et al. Reemerging Leptospirosis, California. Emerg Infect Dis 2004; 10 (3): 406-12.

20.- McDonough P L. Leptospirosis en caninos estado actual. 2001. Disponible en: http://www. ivis.org/advances/Infect_Dis_Carmichael/ mcdonough/ivis.pdf (Acceso el 21 de marzo de 2011).

21.- Michel V, Branger C, Andre-Fontaine G. Epidemiology of leptospirosis. Rev Cubana de Med Trop 2002; 54 (1): 7-10.

22.- Pappas G, Papadimitriou P, Siozopoulou V, Christou L, Akritidis N. The globalization of leptospirosis: worldwide incidence trends. Int J Infect Dis 2008; 12: 351-7.

23.- Hartskeerl R, Collares-Pereira M, Ellis W. Emergence, control and re-emerging leptospirosis: dynamics of infection in the changing world. Clin Microbiol Infect 2011; 17 (4): 494-501.

24.- Coelho M, Massad E. The impact of climate on Leptospirosis in São Paulo, Brazil. Int J Biometeorol 2012; 56 (2): 233-41.

25.- Perret P, Abarca V, Dabanch P, Solari G, García C, Carrasco L, et al. Risk factors and frequency of positive antibodies for leptospirosis in a suburban population near Santiago. Rev Med Chile 2005; 133 (4): 426-31.

26.- Olea A. Enfermedades de notificación obligatoria: zoonosis. Boletín de vigilancia en salud pública El Vigía 2003; 7 (19): 39-44.

27.- Arias H, Núñez M, Valenzuela I, Olivares A. Brote epidémico de leptospirosis en niños de Linares. Rev Chilena Pediatr 2003; 74 (4): 405-10.

28.- Riedemann S, Zamora J. Aspectos epidemiológicos de leptospirosis humana en el medio rural. Zentralblatt für Veterinärmedizin Reihe B 1982; 29 (9): 702-7.

29.- Lobos C. Prevalencia de anticuerpos a serovares del género Leptospira en perros vagos de Chillán mediante el test de microaglutinación (MAT). Memoria de título Médico Veterinario, Universidad de Concepción, Facultad de Ciencias Agropecuarias y Forestales, Departamento de Medicina Veterinaria, Concepción, Chile. 2008.

30.- Sothers R. Prevalencia de leptospirosis en perros de la ciudad de Chillán. Memoria de título Médico Veterinario, Universidad de Concepción, Facultad de Medicina Veterinaria, Departamento de Patología y Medicina Preventiva, Concepción, Chile. 1997.

31.- García M. Determinación de la frecuencia de leptospirosis en perros urbanos de la ciudad de Chillán, Ñuble, por el Test de Microaglutinación. Memoria de título de Médico Veterinario, Universidad de Concepción, Facultad de Ciencias Agropecuarias y Forestales, Departamento de Medicina Veterinaria, Concepción, Chile. 1987.

32.- Zamora J, Kruze J, Riedemann S. Leptospirosis in domestic animals in the south of Chile. Serological study. Zentralbl Veterinar Med B 1975; 22 (7): 544-55.

33.- Thrusfield M. Veterinary epidemiology. 2ed. Oxford: Blackwell Science 1995.

34.- Biogal Galed Laboratories. Canine Leptospira Antibody Test Kit (Intruction manual). 2008. Disponible en: http://biogal.co.il/wp-content/ uploads/2012/01/MICLC311.pdf (acceso el 15 de abril de 2011).

35.- García RL, Machado H, Abeledo Ma A, Feraud D. Utilización de una técnica serológica rápida para el diagnóstico de la leptospirosis canina. REDVET 2009; 10 (7): 1-9.

36.- Carrada-Bravo T. Leptospirosis humana: historia natural, diagnóstico y tratamiento. Rev Mex Patol Clin 2005; 52 (4): 246-56.

37.- De Aguirre L, Jelambi F. Métodos de Diagnóstico de la Leptospirosis, FONAIAP DIVULGA 1991. Disponible en: http://sian.inia. gob.ve/repositorio/revistas_tec/FonaiapDivulga/ fd35/texto/metodos.htm (Acceso el 9 de mayo de 2012).

38.- Sociedad Chilena de Infectología Veterinaria y Comité de Infecciones Emergentes Sociedad Chilena de Infectología. Pauta técnica de vigilancia de enfermedades transmisibles en pequeños animales de compañía. n.d. Disponible en: www.sochipe.cl/subidos/ revista1/docs/PautaTecnica.doc (Acceso el 12 
abril de 2011)

39.- Tilley L, Smith F, MacMurray A. La consulta veterinaria en 5 minutos: canina y felina. $1^{\circ}$ ed. Buenos Aires: Editorial Inter-Médica 1998.

40.- Sepúlveda A, Santiago J, Preciado F J. La rata y el perro, importantes vectores de la leptospirosis en explotaciones pecuarias de Cd. Guzmán, Jalisco. Rev Cubana Med Trop 2002; 54 (1): 21-3.

41.- Greene C. Canine Leptospirosis, emerging or surging?., $59^{\circ}$ Congresso Internazionale Multisala SCIVAC. SCIVAC (società Culturale Italiana Veterinari per Animali da Compagnia), May 30- June 1, 2008. Rimini, Italia.

42.- Rubel D, Seijo A, Cernigoi B, Viale A,
Wisnivesky C. Leptospira en una población canina del Gran Buenos Aires: variables asociadas con la seropositividad. Rev Panam Salud Pública 1997; 2: 102-5.

43.- Pineda M, López J, García M. Frecuencia de leptospirosis en perros al test de aglutinación microscópica en Chillán, Chile. Arch Med Vet 1996; 28 (1): 59-66.

44.- Barrientos J. Contribución al estudio de la leptospirosis canina en el área rural de la provincia de Valdivia. Memoria de título Médico Veterinario, Universidad Austral de Chile, Escuela de Medicina Veterinaria, Valdivia, Chile. 1966.

45.- Rivera A, de la Peña Moctezuma A, Roa M,
Ordoñez M. Seroprevalencia de leptospirosis en perros callejeros del norte de la Ciudad de México. Vet Méx 1999; 30 (1): 105-7.

46.- Rosas P. Frecuencia de leptospirosis canina en dos albergues de Veracruz y Boca del Río, Veracruz, México. Memoria de título de Médico Veterinario, Universidad Veracruzana, Facultad de Medicina Veterinaria y Zootecnia, Veracruz, México. 2011.

47.- Romero M, Sánchez J. Seroprevalencia de la leptospirosis canina de tres municipios del Departamento del Tolima-Colombia. MVZ Córdoba 2009; 14 (2): 1684-9.

48.- Sandow K, Ramírez W. Leptospirosis. REDVET 2005; 6 (6): 1-61. 